\title{
An association study between Heme oxygenase-1 genetic variants and Parkinson's disease
}

\author{
Pedro Ayuso ${ }^{1,2}$, Carmen Martínez ${ }^{2,3}$, Pau Pastor 4,5,6, Oswaldo Lorenzo-Betancor ${ }^{4,5}$, Antonio Luengo ${ }^{3}$, \\ Félix J. Jiménez-Jiménez ${ }^{7}$, Hortensia Alonso-Navarro ${ }^{7}$, José A. G. Agúndez ${ }^{2,3}$ and \\ Elena García-Martín ${ }^{1,2 *}$
}

\footnotetext{
${ }^{1}$ Department of Biochemistry, Molecular Biology, and Genetic, University of Extremadura, Cáceres, Spain

2 Redes Temáticas de Investigación Cooperativa en Salud (RIRAAF/RETICS), Instituto de Salud Carlos III, Madrid, Spain

${ }^{3}$ Department of Pharmacology, University of Extremadura, Cáceres, Spain

${ }^{4}$ Neurogenetics Laboratory, Division of Neurosciences, Center for Applied Medical Research (CIMA), University of Navarra, Pamplona, Spain

${ }^{5}$ Department of Neurology, School of Medicine, Clínica Universidad de Navarra, University of Navarra, Pamplona, Spain

${ }^{6}$ Centro de Investigación Biomédica en Red de Enfermedades Neurodegenerativas (CIBERNED), Instituto de Salud Carlos III, Madrid, Spain

7 Section of Neurology, Hospital Universitario del Sureste, Madrid, Spain
}

Edited by:

Ramon Santos El-Bachá,

Universidade Federal da Bahia, Brazil

Reviewed by:

Jingyu Liu, Mind Research Network, USA

Jie Cui, Roskamp Institute, USA

*Correspondence:

Elena García-Martín, Department of Biochemistry, Molecular Biology,

and Genetics, University of

Extremadura, Avda. de la

Universidad s/n, 10071-Cáceres,

Spain

e-mail: elenag@unex.es
The blood-brain barrier (BBB) supplies brain tissues with nutrients, filters harmful compounds from the brain back to the bloodstream, and plays a key role in iron homeostasis in the human brain. Disruptions of the BBB are associated with several neurodegenerative conditions including Parkinson's disease (PD). Oxidative stress, iron deposition and mitochondrial impaired function are considered as risk factors for degeneration of the central nervous system. Heme oxygenase (HMOX) degrades heme ring to biliverdin, free ferrous iron and carbon monoxide being the rate-limiting activity in heme catabolism. The isoform HMOX1 is highly inducible in response to reactive oxygen species, which induce an increase in BBB permeability and impair its pathophysiology. Consequently, an over- expression of this enzyme may contribute to the marked iron deposition found in PD. We analyzed the HMOX1 SNPs rs2071746, rs2071747, and rs9282702, a microsatellite $(\mathrm{GT})_{n}$ polymorphism and copy number variations in 691 patients suffering from PD and 766 healthy control individuals. Copy number variations in the HMOX1 gene exist, but these do not seem to be associated with PD risk. In contrast two polymorphisms that modify the transcriptional activity of the gene, namely a VNTR $(\mathrm{GT})_{n}$ and the SNP rs2071746, are strongly associated with PD risk, particularly with the classic PD phenotype and with early onset of the disease. This study indicates that HMOX1 gene variants are associated to the risk of developing some forms of PD, thus adding new information that supports association of HMOX gene variations with PD risk.

Keywords: Parkinson's disease, Heme oxygenase, polymorphisms, copy number variations, biomarkers, blood-brain barrier, iron homeostasis

\section{INTRODUCTION}

The blood-brain barrier (BBB) supplies brain tissues with nutrients, filters harmful compounds from the brain back to the bloodstream, and plays a key role in iron homeostasis in human brain. Disruptions of the BBB are associated with several neurodegenerative diseases including Parkinson's disease (PD). Oxidative stress, iron deposition and mitochondrial impaired function are considered as risk factors for degeneration of the central nervous system (Schipper, 2004; Alonso-Navarro et al., 2008). In addition, it has been postulated that BBB inflammation may be related to PD risk by causing over-activation of microglia, increased production of cytokines and release of reactive oxygen species (Whitton, 2007), and the possibility that PD may be related to blood borne toxins could be the cause of these neurodegenerative disorders if the integrity of $\mathrm{BBB}$ is compromised (for a recent review, see Kim et al., 2012).
$\mathrm{PD}$ is one of the most common neurodegenerative movement disorders; current demographic trends indicate a lifetime risk approaching 4\% (Schapira, 2013). The mean age of onset is 70 years, although $4 \%$ of patients develop early-onset, before the age of 50 (Schrag and Schott, 2006). This disease is characterized by rest tremors, rigidity, slowness of movement and postural imbalance. The primary pathologic abnormalities are the loss of the pigmented cells of the substancia nigra pars compacta, and the dopaminergic neurons of the striatum, which decreases in dopamine levels (Lu'o'ng and Nguyen, 2012). Other central and peripheral dopaminergic and non-dopaminergic systems are also involved. Regarding the pathogenesis of neurodegeneration in PD, patients show increased levels of oxidized lipids and a decrease in levels of glutathione (Zeevalk et al., 2008; Obeso et al., 2010; Schapira, 2012). In these patients, concentration of phospholipids and polyunsaturated free fatty acids, which are highly susceptible to oxidants, is decreased, whereas malondialdehyde, a 
marker of lipid oxidation, is increased (Dexter et al., 1989; Zhou et al., 2008). Several studies have suggested the occurrence of genetic factors related to PD risk, but so far no major genetic risk factors have been identified, and it is widely accepted that PD is likely to be related to genetic plus environmental interaction (Schrag and Schott, 2006; Obeso et al., 2010; Trinh and Farrer, 2013; Alonso-Navarro et al., 2014) and that further genetic association studies based on solid functional mechanisms related to the pathogenesis of the disease are required.

A relevant feature of the development of PD is abnormal iron deposition in the substantia nigra of PD patients, as has been demonstrated both in post-mortem studies (Sofic et al., 1988) and in in vivo studies (Hochstrasser et al., 2004; Berg and Hochstrasser, 2006). Some consequences of elevated nigral iron levels are well known. The intranigral administration of iron produces selective dopaminergic neuronal loss and elicits parkinsonian symptoms (Youdim, 2003). Alteration of iron homeostasis, dysfunction in molecules involved in sequestering excess iron, as well as ageing, are considered as possible causes of elevated iron deposition. BBB protects the brain against circulating iron, however the specific mechanisms involved in the regulation of iron release and transport that lead to iron accumulation in aging and neurological disorders such as PD, are not completely understood. The involvement of dysregulation of brain iron homeostasis in PD has been recently revised (Weinreb et al., 2013). It was suggested that alterations in brain vascularization in certain areas may cause high levels of iron (Faucheux et al., 1999).

Heme oxygenase (HMOX) enzyme activity degrades heme ring to biliverdin, free ferrous iron and carbon monoxide being the rate-limiting activity in heme catabolism (Ryter and Tyrrell, 2000). Two HMOX isoforms have been described in the mammalian central nervous system: HMOX1 is present in neurons and neuroglia (Benjamini and Hochberg, 1995), and it is inducible or repressible by pro-oxidant and other microenvironment stimuli (Kinobe et al., 2006; Loboda et al., 2008). HMOX2 is ubiquitously and constitutively expressed throughout the mammalian neuraxis, predominantly in substantia nigra, septum and hippocampus (Verma et al., 1993; Maines, 1997). A third isoform unique to rats, designated as HMOX3, exists (Scapagnini et al., 2002). The role of single nucleotide polymorphisms (SNPs) in the HMOX2 gene in patients with PD has been reported, the HMOX2 SNP rs2270363 being associated with PD risk (Ayuso et al., 2011). In contrast, little is known about the role of genetic variations in the HMOX1 gene in PD, and controversial findings justify the analysis of this gene in a large sample population (Funke et al., 2009; Infante et al., 2010). HMOX1 is a plausible candidate gene for PD risk because immunoreactive HMOX1 has been identified in dopaminergic neurons of PD patients (Castellani et al., 1996; Schipper, 1998). In addition, neurotoxins, cytokines and nitric oxide are considered as inducers of astroglial HMOX1 expression (Rieder et al., 2004; Schipper, 2004). Moreover, under stress conditions in astroglia, HMOX1 promotes mitochondrial sequestration of non-transferrin iron and may contribute to the pathological iron deposition described in PD (Schipper et al., 2009). In this study we analyze common variations and copy number variations in the HMOX1 gene, selected according to their allelic frequencies and putative functional effect on enzyme activity, in a large sample of patients with PD and healthy controls.

\section{METHODS}

Subjects were recruited from several centers in Spain. The study group was composed of $691 \mathrm{PD}$ patients and 766 healthy controls, which were included in the study after obtaining informed consent. From these, 460 patients with PD and 459 control individuals were recruited from the Clínica Universidad de Navarra (Pamplona, Spain). In addition, 231 patients with PD were recruited from the Hospital de La Princesa (Madrid, Spain) and 307 controls were included from the latter hospital and from the Infanta Cristina University Hospital (Badajoz, Spain). Most participants had been included in previous studies by our group (Agundez et al., 2008a; Garcia-Martin et al., 2008; Ayuso et al., 2011; Lorenzo-Betancor et al., 2011; Jimenez-Jimenez et al., 2014). All the participants were Caucasian Spanish individuals as stated in the self-report. All consecutive patients diagnosed by consultant neurologists according to the criteria recommended by Hughes et al. (1992) were requested to participate and all of them agreed to do so. Selection criteria for patients with PD included bradykinesia and at least one of the following symptoms: rigidity, resting tremor, postural instability, a positive response to dopaminergic therapy, and the absence of atypical features or other causes of parkinsonism (Hughes et al., 1992). From the total of patients, only 36 of the 691 patients with PD had a positive family history of the disease and monogenic forms of PD were excluded. Patients were divided in three groups according to different phenotypes: tremor-dominant patients with PD (TD-PD), classical phenotype patients with PD (C-PD), or akinetic-rigid patients with PD (AR-PD) when such information was available (Lorenzo-Betancor et al., 2011). As the classification of motor PD phenotype subgroups can be arbitrary, we considered those individuals with rest tremor as dominant and an initial feature of their disease to have a TD-PD. AR-PD phenotype was considered for those individuals with predominant bradykinesia or signs of rigidity, but mild or no tremor at rest. The patients with comparable severity of tremor, rigidity, and bradykinesia were considered patients with C-PD. A medical examination was performed to identify individuals in good health. The study was approved by the ethics committees of the institutions involved in the study. For all participants, genomic DNA was obtained from peripheral leukocytes and purified according to standard procedures. Table 1 summarizes the demographic data of the subgroups analyzed in the study.

Four polymorphisms in the HMOX1 gene, located in chromosome 22q12, were selected for genotyping on the basis of putative functional effects as well as expected allele frequency in Caucasian individuals as reported in public databases (http://www.ncbi.nlm.nih.gov/projects/SNP/snp_ref. cgi? showRare $=$ on $\&$ chooseRs $=$ all $\& g o=$ Go\&locusId $=3162)$. These polymorphisms included a variable number tandem repeat (VNTR), consisting of alternating the purine-pyrimidine sequence $(\mathrm{GT})_{n}$ which has the potential to acquire the Z-DNA conformation, a structure which is thermodynamically unfavorable compared with the B-DNA structure. This formation has been described as a factor that negatively affects transcriptional 
Table 1 | Demographic data of the sample analyzed in this study.

\begin{tabular}{|c|c|c|c|c|c|c|}
\hline Group & Controls $(n=766)$ & \multicolumn{5}{|c|}{ PD } \\
\hline Age, y, mean $(S D)$ & 64.67 (14.28) & $67.09(10.62)$ & 69,51 (8.09) & $67.06(10.68)$ & $66.00(10.79)$ & $67.32(11.76)$ \\
\hline Age range, y & 17-102 & 22-95 & 49-87 & 22-95 & 39-87 & 41-88 \\
\hline$A A O, y$, mean $(S D)$ & NA & $59.57(12.61)$ & $62.82(8.13)$ & 57.55 (11.58) & 56.81 (11.59) & $63.51(13.65)$ \\
\hline Female \% & $45.7 \%$ & $42.6 \%$ & $43.3 \%$ & $36.8 \%$ & $42.5 \%$ & $50.2 \%$ \\
\hline
\end{tabular}

$P D$, Parkinson's disease; $y$, years; $A A O$, age at onset; $S D$, standard deviation; NA, not available; TD-PD, tremor-dominant Parkinson's disease; $C-P D$, classical $P D$ phenotype; $A R-P D$, Akinetic-rigid $P D$.

${ }^{a} P D$ subjects with no information on motor features available.

activity (Naylor and Clark, 1990; Delic et al., 1991) and that causes differences in transcriptional activity of the promoter of $H M O X 1$ with different numbers of repeats. For the association study of the HMOX1 $\mathrm{GT}_{(n)}$, the alleles were divided into two classes: class $\mathrm{S}$ including short repeat sequences $(n<25)$ and class $\mathrm{L}$ including long repeat sequences $(n \geq 25)$ (Yamada et al., 2000; Tiroch et al., 2007). The presence of this VNTR polymorphism was analyzed after PCR amplification (forward primer $5^{\prime}$-AGAGCCTGCAGCTTCTCAGA-3' and reverse primer $5^{\prime}$-CTCTGGCTTCCTAGCAGGG-3') and quantification of number of repetitions by using GeneGel HyRes Denaturing gels, and DNA staining kit Silver staining kit (GE Healthcare, Madrid, Spain).

Three single nucleotide polymorphisms were studied by means of TaqMan probes (Applied Biosciences Hispania, Alcobendas, Madrid, Spain): The SNP rs2071746 (A/T) is an upstream variant located at 22:35380679, rs2071747 is a missense mutation G/C (Asp/His) located at 22: 35381192, which corresponds to exon 1 of the HMOX1 gene, and rs9282702 consists of a missense mutation C/T (Pro/Leu) located at 22:35386857, which corresponds to exon 3 of the HMOX1 gene. Besides these SNPs, no other non-synonymous HMOX1 SNPs have been reported to occur in Caucasian individuals at frequencies over $1 \%$. Custom primers were designed to analyze the SNPs rs9282702 and rs2071747, whereas commercial primers were used for the detection of the SNPs rs2071746 (C__15869717_10, Applied Biosciences Hispania, Alcobendas, Madrid, Spain). The detection was carried out by qPCR in an Eppendorf Realplex thermocycler by using fluorescent probes. The amplification conditions were as follows: After a denaturation time of $10 \mathrm{~min}$ at $96^{\circ} \mathrm{C}, 45$ cycles of $92^{\circ} \mathrm{C} 15 \mathrm{~s} 60^{\circ} \mathrm{C} 90 \mathrm{~s}$ were carried out and fluorescence was measured at the end of each cycle and at endpoint. All samples were determined in triplicate and genotypes were assigned both by gene identification software (RealPlex 2.0, Eppendorf) and by analysis of the reference cycle number for each fluorescence curve, calculated by the CalQPlex algorithm (Eppendorf). For technical validation purposes, the amplified fragments for 20 individuals carrying every genotype (AA, AT and TT for rs2071746 and GG, GC and all carriers of the CC genotype for rs2071747 were sequenced, and in all cases the genotypes fully corresponded with those detected with fluorescent probes.
Copy number variations (CNVs) of the HMOX1 gene were analyzed using the TaqMan copy number assay Hs00774483_cn (Applied Biosciences Hispania), which was designed to hybridize within the open reading frame in HMOX1 gene exon 3 (chromosome location 22:35782935). Amplification was carried out in an Applied Biosystems 7500 real-time thermocycler as described by the manufacturer, using as a copy number reference assay RNAse P. All reactions were carried out in quadruplicate. Results were analyzed by means of the CopyCaller Software (Applied Biosciences Hispania). According to standard procedures in CNV analyses, we designed as heterozygous (null/present) those samples with a single copy of the HMOX1 gene.

\section{STATISTICAL ANALYSIS}

The Hardy-Weinberg equilibrium was analyzed with the DeFinetti program (http://ihg.gsf.de/cgi-bin/hw/hwa1.pl). Allelic and genotype analyses were performed with the PLINK software (Purcell et al., 2007). Haplotype reconstruction was performed using the program PHASE v2.1.1 (Stephens et al., 2001). This reconstruction was carried out to detect putative association of at risk haplotypes. We used the default model for recombination rate variation with 1000 iterations, 500 burn-in iterations, and a thinning interval of 1 . Diplotypes were obtained from the combination of haplotypes in the best run (the one that showed the maximum consistency of results across all runs); further details are provided elsewhere (Agundez et al., 2008b). Statistical analyses were performed using the SPSS 15.0 for Windows (SPSS Inc., Chicago, Illinois, USA). Intergroup comparison values were calculated by using the $\chi^{2}$ or Fisher tests when appropriate. The $95 \%$ confidence intervals were also calculated. The threshold for statistical significance was $p<0.05$. Correction for multiple testing was done according the false discovery rate (FDR) procedure as described elsewhere (Benjamini and Hochberg, 1995). The statistical power was determined from variant allele frequencies observed in control individuals with a genetic model analyzing the frequency for carriers of the disease gene with a relative risk $(\mathrm{RR})$ value $=1.5(p=0.05)$. The statistical power for two-tailed associations for the presence of any of the two SNPs identified in this study was $99.9 \%$ for overall patients with PD, $98.3 \%$ for patients with C-PD and $95.4 \%$ for patients with 
an unknown PD phenotype. Testing for heterogeneous association (homogeneity test) was analyzed by using the Breslow-Day test.

\section{RESULTS}

At the first stage, because of DNA shortage, we analyzed the occurrence of the four HMOX1 gene polymorphisms, including the VNTR and the three SNPs described under methods, in 100 randomly selected samples from PD patients and 150 samples from healthy individuals. The SNP rs9282702 was monomorphic. The rest of gene variations analyzed were found to be polymorphic in the population study, and therefore we extended the analyses to all patients and control individuals participating in the study. CNV analyses in the whole study group revealed the occurrence of three patients with PD and six control individuals with a single copy of the HMOX1 gene. All these individuals were carriers of one null allele in heterozygosity. Individuals with zero or more than two gene copies were not identified in the study group. To our knowledge this is the first description of the occurrence of CNVs in the HMOX1 gene, but the occurrence of CNVs does not seem to have a major association with PD risk.

The combined genotypes obtained after SNP and CNV analyses are summarized in Table 2. No deviation from HardyWeinberg equilibrium was found for the SNPs or CNVs analyzed either in the subgroups of patients suffering from PD or in the control group. The risk of PD associated with the SNPs and CNVs was estimated considering the major allele as the risk allele, by comparison of allelic, heterozygous, and homozygous dominant and recessive models. The major alleles for the SNPs rs2071746 and rs2071747 were A and G, respectively, in agreement with the allele frequencies shown in the 1000 genomes catalog for European individuals (http://browser.1000genomes. org/Homo_sapiens/Info/Index). The best fit was observed with the dominant and the allelic models. We identified a statistically significant difference of the rs2071746 genotypes between patients with C-PD and healthy individuals. This difference was observed both in the dominant model and in the allelic model. After correction for the three PD presentations (TD$\mathrm{PD}, \mathrm{C}-\mathrm{PD}$, and AR-PD), these associations remained statistically significant in the C-PD group. In addition, a weak statistically significant difference in the frequency of HMOX1 rs2071746 genotypes between patients with TD-PD and healthy individuals was observed. This difference followed a dominant model, but the difference was not statistically significant in the allelic model (Table 2). When the 20 patients with C-PD and positive family history of PD were excluded, the statistical significance of the OR values were similar (not shown). No association with the SNP rs2071747 was identified in any of the PD phenotypes. The SNPs, rs2071746, and rs2071747 did not show linkage disequilibrium $\left(D^{\prime}=0.652, r=0.147\right)$, and no particular risk haplotypes were identified in any of the study groups.

As has been described previously, patients with the phenotypes TP-PD and C-PD may have an earlier age at the onset than the AR-PD group (Rajput et al., 2009), so we analyzed whether HMOX1 rs2071746 genotype frequencies were related to age of

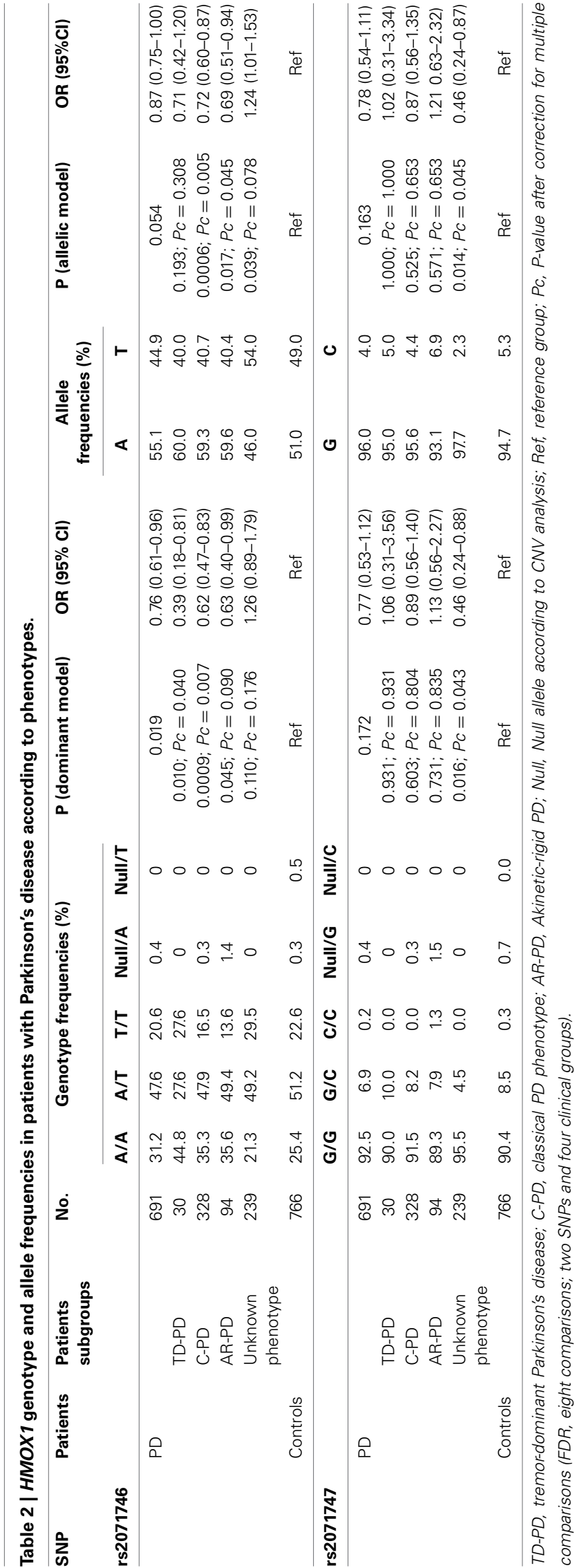


onset in the C-PD subgroup. Patients with C-PD phenotype were subdivided according to the median age at onset in this subgroup (62 years). Table 3 summarizes these results. We observed statistically significant differences among patients with early onset of C-PD both, in the dominant model and in the allelic model, whereas such significant differences were not observed among patients with late-onset C-PD.

Regarding the HMOX-1 $\mathrm{GT}_{(n)}$ polymorphism, we studied a subset of the total of patients and healthy subjects included in this study because of DNA shortage. Thus, 365 PD patients and 371 healthy subjects were genotyped. The number of repeats ranged from 11 to 33, with the highest frequency for $\mathrm{GT}_{(20)}$ repeats in healthy subjects and $\mathrm{GT}_{(22)}$ repeats in the case of $\mathrm{PD}$ patients. Mann-Whitney comparison for means in each group revealed statistically significant differences $(p<0.001)$. The association study of this polymorphism with PD patients is shown Table 4.

\section{DISCUSSION}

BBB permeability is increased in the presence of reactive oxygen species, and it is widely accepted that the HMOX1 mRNA upregulation in CNS is a marker of oxidative stress. The enhanced expression of HMOX1 may contribute to the pathological deposition of iron which has been reported in normal aging CNS, and to a much greater extent in neurodegenerative disorders such as PD (Droge, 2002). Impaired HMOX1 activity caused by genetic variants could modify the intraneuronal production of biliverdin/bilirubin metabolites which exert an antioxidant role (Dore et al., 1999) contributing to the etiology of PD and other central nervous system disorders. Our group reported that functional variations in $H M O X 2$ gene are associated with PD risk (Ayuso et al., 2011). In this context and bearing in mind that HMOX1 has been postulated as a contributor to the pathological iron deposition described in PD (Schipper et al., 2009), we aimed to analyzed the possible role of $H M O X 1$ genetic variants in a large series of PD patients.

We identified a clinical association of the HMOX1 rs2071746 polymorphism (A/T) with C-PD. The rs2071746 A allele was more common among PD patients with C-PD phenotype than in control individuals. Also, we observed that this association was related with an early onset of the disease. In addition, the analysis of the length of the highly polymorphic $(\mathrm{GT})_{n}$ dinucleotide repeats indicates an association of longer $(\mathrm{GT})_{n}$ repeats with susceptibility to PD. Both gene variations, rs2071746 and $(\mathrm{GT})_{n}$ dinucleotide repeats sequence, have been related to variations in the transcriptional activity of the HMOX1 gene (Yamada et al., 2000; Tanaka et al., 2011; Kramer et al., 2013). According published evidence, the number of $(\mathrm{GT})_{n}$ repeats inversely correlates with HMOX1 promoter activity and inducibility of the gene (Yamada et al., 2000), although controversy exist (Tanaka et al., 2011). It has been shown that the rs2071746 A allele is associated with high promoter activity (Ono et al., 2004). Our findings support an increased frequency of carriers of long $(\geq 25$ $(\mathrm{GT})_{n}$ ) among PD patients and an increased frequency of carriers of the rs2071746 A allele among PD patients. Although these findings apparently point to opposite directions in terms of gene activity, the functional effect of these polymorphisms is not fully

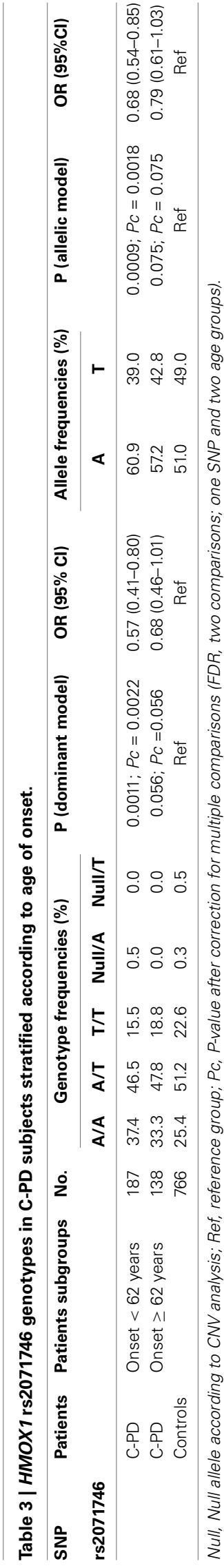


Table 4 | Genotype and allele frequencies for (GT) $n_{n}$ repeat polymorphisms in PD patients and healthy subjects.

\begin{tabular}{|c|c|c|c|c|c|c|c|c|c|}
\hline \multirow[t]{2}{*}{ Patients } & \multicolumn{3}{|c|}{ Genotype frequencies (\%) } & \multirow[t]{2}{*}{ P (dominant model) } & \multirow[t]{2}{*}{ OR $(95 \% \mathrm{Cl})$} & \multicolumn{2}{|c|}{ Allele frequencies (\%) } & \multirow[t]{2}{*}{ P (allelic model) } & \multirow[t]{2}{*}{ OR $(95 \% \mathrm{Cl})$} \\
\hline & S/S & $S / L$ & $\mathbf{L} / \mathbf{L}$ & & & $\mathbf{S}$ & $\mathbf{L}$ & & \\
\hline $\mathrm{PD}(N=365)$ & 86.3 & 12.3 & 1.4 & 0.0001 & $0.34(0.20-0.59)$ & 0.92 & 0.08 & 0.0001 & $0.37(0.23-0.62)$ \\
\hline Controls $(N=371)$ & 94.9 & 4.3 & 0.8 & & & 0.97 & 0.03 & Ref & \\
\hline
\end{tabular}

$S$, short repeat sequences $(n<25(G T) n) ; L$ long repeats sequences $(n \geq 25(G T) n)$.

understood. Moreover, an extended model of the HMOX1 gene along with the importance of alternative splicing in the area where these two polymorphisms are located, and its association with translational regulation has been disclosed recently (Kramer et al., 2013).

Our results agree with those by Kimpara et al. (1997) although other studies that failed to confirm these findings have been published (Funke et al., 2009). Putative discrepancy in the genetic associations may be explained because of the different ethnic origin of the participants included in each of these studies. Infante et al. (2010) reported association of the rs2071746 $\mathrm{T}$ allele in homozygosity as a risk factor for PD, but only when combined with another SNPs at the GSK3beta gene, and in a relatively small study group. In the study by Infante et al. however the rs 2071746 $\mathrm{T}$ allele frequency is almost identical in patients and controls (39 and $37 \%$ ). In fact, when the comparison of the rs2071746 genotypes or allele frequencies between patients and controls is made, no statistically significant differences are observed (Infante et al., 2010). In our study the association is statistically significant when analyzing genotypes or allele frequencies. Moreover, the large sample size analyzed in the present study and the fact that positive associations remained statistically significant after correction for multiple testing support the observed genetic associations. The effect of the rs2071746 SNP on different PD subgroups seems presenting consistent patterns, though some did not pass significance threshold. In fact, taking into consideration the dominant model (Table 2) the findings obtained in TD-PD and C-PD patients show different directions. Nevertheless it should be considered that the subgroup of TD-PD is composed of 30 patients only. These apparent discrepancies, and the lack of statistical significance in some subgroups, may be related to the small sample size of some of these subgroups. In addition, we analyzed for the first time the occurrence of CNVs in HMOX1, both in PD patients and in healthy individuals. We found CNVs more frequently in AR-PD patients. Although this study does not support a major role of $H M O X 1 \mathrm{CNV}$ s in PD risk, the demonstration of the occurrence of CNVs in the HMOX1 gene supports further research in the functional role and clinical associations of HMOX1 CNVs. It is of note, however, that the association cannot be observed in the subgroup of PD patients with unknown phenotype. Our findings suggest that the association of HMOX1 genotypes and PD risk is specific of the C-PD phenotype. This reinforces the need of detailed phenotyping and categorization of patients in genetic association studies, particularly when assessing low-penetrance factors. In summary, in this study we identified two functional HMOX1 gene variations as potential genetic biomarkers of PD. It is conceivable that these genetic biomarkers would be associated to other disorders related to oxidative stress in the CNS and in the BBB. This study adds to current knowledge of genetic biomarkers in PD (Alonso-Navarro et al., 2014) and other CNS disorders (Agundez et al., 2013) and, once these biomarkers are validated, these could be used to identify at risk individuals and to provide proof of concept regarding the mechanisms involved in the development of these diseases. Further research would be needed to analyze $H M O X 1$ genetic variations in an independent population to verify these associations and to analyze putative interethnic variability in the observed genetic association.

\section{ACKNOWLEDGMENTS}

We thank all the participating subjects for their collaboration. We are grateful to Prof. James McCue for assistance in language editing. Research at author's laboratories is financed by grants PS09/00943, PS09/00469, PI12/00241, PI12/00324, and RETICS RD12/0013/0002 from Fondo de Investigación Sanitaria, Instituto de Salud Carlos III, Madrid, Spain; SAF2006-10126: and SAF2010-22329-C02-01; GR10068 from Junta de Extremadura, Spain; 061131 from the "Fundació La Marató de TV3" and by the UTE project FIMA. Financed in part with FEDER funds from the European Union.

\section{REFERENCES}

Agundez, J. A., Golka, K., Martinez, C., Selinski, S., Blaszkewicz, M., and GarciaMartin, E. (2008b). Unraveling ambiguous NAT2 genotyping data. Clin. Chem. 54, 1390-1394. doi: 10.1373/clinchem.2008.105569

Agundez, J. A., Jimenez-Jimenez, F. J., Alonso-Navarro, H., and Garcia-Martin, E. (2013). Genetic biomarkers of essential tremor: time to think outside of the box. Pharmacogenomics 14, 1681-1682. doi: 10.2217/pgs.13.168

Agundez, J. A., Luengo, A., Herraez, O., Martinez, C., Alonso-Navarro, H., JimenezJimenez, F. J., et al. (2008a). Nonsynonymous polymorphisms of histaminemetabolising enzymes in patients with Parkinson's disease. Neuromolecular Med. 10, 10-16. doi: 10.1007/s12017-007-8017-7

Alonso-Navarro, H., Jimenez-Jimenez, F. J., Garcia-Martin, E., and Agundez, J. A. (2014). Genomic and pharmacogenomic biomarkers of Parkinson's disease. Curr. Drug Metab. 15, 129-181. doi: 10.2174/138920021502140327175404

Alonso-Navarro, H., Jiménez-Jiménez, F. J., Pilo-de-la-Fuente, B., and PlazaNieto, J. F. (2008). "Mecanismos patogénicos de la enfermedad de Parkinson," in Tratado de los Trastornos del Movimiento, eds F. J. Jiménez-Jiménez, M. R. Luquin, J. A. Molina, and G. Linazasoro (Barcelona: Viguera Editores), 425-485.

Ayuso, P., Martinez, C., Lorenzo-Betancor, O., Pastor, P., Luengo, A., JimenezJimenez, F. J., et al. (2011). A polymorphism located at an ATG transcription start site of the heme oxygenase- 2 gene is associated with classical Parkinson's disease. Pharmacogenet. Genomics 21, 565-571. doi: 10.1097/FPC.0b013e328348f729

Benjamini, Y., and Hochberg, Y. (1995). Controlling the false discovery rate: a practical and powerful approach to multiple testing. J. R. Stat. Soc. Ser. B 1, 289-300.

Berg, D., and Hochstrasser, H. (2006). Iron metabolism in Parkinsonian syndromes. Mov. Disord. 21, 1299-1310. doi: 10.1002/mds.21020

Castellani, R., Smith, M. A., Richey, P. L., and Perry, G. (1996). Glycoxidation and oxidative stress in Parkinson disease and diffuse Lewy body disease. Brain Res. 737, 195-200. doi: 10.1016/0006-8993(96)00729-9 
Delic, J., Onclercq, R., and Moisan-Coppey, M. (1991). Inhibition and enhancement of eukaryotic gene expression by potential non-B DNA sequences. Biochem. Biophys. Res. Commun. 181, 818-826. doi: 10.1016/0006291X(91)91263-C

Dexter, D. T., Carter, C. J., Wells, F. R., Javoy-Agid, F., Agid, Y., Lees, A., et al. (1989). Basal lipid peroxidation in substantia nigra is increased in Parkinson's disease. J. Neurochem. 52, 381-389. doi: 10.1111/j.1471-4159.1989.tb09133.x

Dore, S., Takahashi, M., Ferris, C. D., Zakhary, R., Hester, L. D., Guastella, D., et al. (1999). Bilirubin, formed by activation of heme oxygenase-2, protects neurons against oxidative stress injury. Proc. Natl. Acad. Sci. U.S.A. 96, 2445-2450. doi: 10.1073/pnas.96.5.2445

Droge, W. (2002). Free radicals in the physiological control of cell function. Physiol. Rev. 82, 47-95. doi: 10.1152/physrev.00018.2001

Faucheux, B. A., Bonnet, A. M., Agid, Y., and Hirsch, E. C. (1999). Blood vessels change in the mesencephalon of patients with Parkinson's disease. Lancet 353, 981-982. doi: 10.1016/S0140-6736(99)00641-8

Funke, C., Tomiuk, J., Riess, O., Berg, D., and Soehn, A. S. (2009). Genetic analysis of heme oxygenase-1 (HO-1) in German Parkinson's disease patients. J. Neural Transm. 116, 853-859. doi: 10.1007/s00702-009-0237-6

Garcia-Martin, E., Ayuso, P., Luengo, A., Martinez, C., and Agundez, J. A. (2008). Genetic variability of histamine receptors in patients with Parkinson's disease. BMC Med. Genet. 9:15. doi: 10.1186/1471-2350-9-15

Hochstrasser, H., Bauer, P., Walter, U., Behnke, S., Spiegel, J., Csoti, I., et al. (2004). Ceruloplasmin gene variations and substantia nigra hyperechogenicity in Parkinson disease. Neurology 63, 1912-1917. doi: 10.1212/01.WNL.0000144276.29988.C3

Hughes, A. J., Daniel, S. E., Kilford, L., and Lees, A. J. (1992). Accuracy of clinical diagnosis of idiopathic Parkinson's disease: a clinico-pathological study of 100 cases. J. Neurol. Neurosurg. Psychiatr. 55, 181-184. doi: 10.1136/jnnp. 55.3 .181

Infante, J., Garcia-Gorostiaga, I., Sanchez-Juan, P., Sierra, M., Martin-Gurpegui, J. L., Terrazas, J., et al. (2010). Synergistic effect of two oxidative stress-related genes (heme oxygenase-1 and GSK3beta) on the risk of Parkinson's disease. Eur. J. Neurol. 17, 760-762. doi: 10.1111/j.1468-1331.2009.02908.x

Jimenez-Jimenez, F. J., Alonso-Navarro, H., Garcia-Martin, E., and Agundez, J. A. (2014). COMT gene and risk for Parkinson's disease: a systematic review and meta-analysis. Pharmacogenet. Genomics 24, 331-339. doi: 10.1097/FPC.0000000000000056

Kim, S. Y., Buckwalter, M., Soreq, H., Vezzani, A., and Kaufer, D. (2012). Blood-brain barrier dysfunction-induced inflammatory signaling in brain pathology and epileptogenesis. Epilepsia 53(Suppl. 6), 37-44. doi: 10.1111/j.1528-1167.2012.03701.x

Kimpara, T., Takeda, A., Watanabe, K., Itoyama, Y., Ikawa, S., Watanabe, M., et al. (1997). Microsatellite polymorphism in the human heme oxygenase-1 gene promoter and its application in association studies with Alzheimer and Parkinson disease. Hum. Genet. 100, 145-147. doi: 10.1007/s004390050480

Kinobe, R. T., Vlahakis, J. Z., Vreman, H. J., Stevenson, D. K., Brien, J. F., Szarek, W. A., et al. (2006). Selectivity of imidazole-dioxolane compounds for in vitro inhibition of microsomal haem oxygenase isoforms. Br. J. Pharmacol. 147, 307-315. doi: 10.1038/sj.bjp.0706555

Kramer, M., Sponholz, C., Slaba, M., Wissuwa, B., Claus, R. A., Menzel, U., et al. (2013). Alternative $5^{\prime}$ untranslated regions are involved in expression regulation of human heme oxygenase-1. PLoS ONE 8:e77224. doi: 10.1371/journal.pone. 0077224

Loboda, A., Jazwa, A., Grochot-Przeczek, A., Rutkowski, A. J., Cisowski, J., Agarwal, A., et al. (2008). Heme oxygenase- 1 and the vascular bed: from molecular mechanisms to therapeutic opportunities. Antioxid. Redox Signal. 10, 1767-1812. doi: 10.1089/ars.2008.2043

Lorenzo-Betancor, O., Samaranch, L., Garcia-Martin, E., Cervantes, S., Agundez, J. A., Jimenez-Jimenez, F. J., et al. (2011). LINGO1 gene analysis in Parkinson's disease phenotypes. Mov. Disord. 26, 722-727. doi: 10.1002/mds.23452

Lu'o'ng, K., and Nguyen, L. T. (2012). Thiamine and Parkinson's disease. J. Neurol. Sci. 316, 1-8. doi: 10.1016/j.jns.2012.02.008

Maines, M. D. (1997). The heme oxygenase system: a regulator of second messenger gases. Annu. Rev. Pharmacol. Toxicol. 37, 517-554. doi: 10.1146/annurev.pharmtox.37.1.517

Naylor, L. H., and Clark, E. M. (1990). d(TG)n.d(CA)n sequences upstream of the rat prolactin gene form Z-DNA and inhibit gene transcription. Nucleic Acids Res. 18, 1595-1601. doi: 10.1093/nar/18.6.1595
Obeso, J. A., Rodriguez-Oroz, M. C., Goetz, C. G., Marin, C., Kordower, J. H., Rodriguez, M., et al. (2010). Missing pieces in the Parkinson's disease puzzle. Nat. Med. 16, 653-661. doi: 10.1038/nm.2165

Ono, K., Goto, Y., Takagi, S., Baba, S., Tago, N., Nonogi, H., et al. (2004). A promoter variant of the heme oxygenase- 1 gene may reduce the incidence of ischemic heart disease in Japanese. Atherosclerosis 173, 315-319. doi: 10.1016/j.atherosclerosis.2003.11.021

Purcell, S., Neale, B., Todd-Brown, K., Thomas, L., Ferreira, M. A., Bender, D., et al. (2007). PLINK: a tool set for whole-genome association and population-based linkage analyses. Am. J. Hum. Genet. 81, 559-575. doi: 10.1086/519795

Rajput, A. H., Voll, A., Rajput, M. L., Robinson, C. A., and Rajput, A. (2009). Course in Parkinson disease subtypes: a 39-year clinicopathologic study. Neurology 73, 206-212. doi: 10.1212/WNL.0b013e3181ae7af1

Rieder, C. R., Williams, A. C., and Ramsden, D. B. (2004). Selegiline increases heme oxygenase- 1 expression and the cytotoxicity produced by dopamine treatment of neuroblastoma SK-N-SH cells. Braz. J. Med. Biol. Res. 37, 1055-1062. doi: 10.1590/S0100-879X2004000700015

Ryter, S. W., and Tyrrell, R. M. (2000). The heme synthesis and degradation pathways: role in oxidant sensitivity. Heme oxygenase has both pro- and antioxidant properties. Free Radic. Biol. Med. 28, 289-309. doi: 10.1016/S08915849(99)00223-3

Scapagnini, G., D’Agata, V., Calabrese, V., Pascale, A., Colombrita, C., Alkon, D. et al. (2002). Gene expression profiles of heme oxygenase isoforms in the rat brain. Brain Res. 954, 51-59. doi: 10.1016/S0006-8993(02)03338-3

Schapira, A. H. (2012). Mitochondrial diseases. Lancet 379, 1825-1834. doi: 10.1016/S0140-6736(11)61305-6

Schapira, A. H. (2013). Recent developments in biomarkers in Parkinson disease. Curr. Opin. Neurol. 26, 395-400. doi: 10.1097/WCO.0b013e3283633741

Schipper, H. M. (1998). Experimental induction of corpora amylacea in adult rat brain. Microsc. Res. Tech. 43, 43-48.

Schipper, H. M. (2004). Brain iron deposition and the free radical-mitochondrial theory of ageing. Ageing Res. Rev. 3, 265-301. doi: 10.1016/j.arr.2004.02.001

Schipper, H. M., Song, W., Zukor, H., Hascalovici, J. R., and Zeligman, D. (2009). Heme oxygenase- 1 and neurodegeneration: expanding frontiers of engagement. J. Neurochem. 110, 469-485. doi: 10.1111/j.1471-4159.2009.06160.x

Schrag, A., and Schott, J. M. (2006). Epidemiological, clinical, and genetic characteristics of early-onset parkinsonism. Lancet Neurol. 5, 355-363. doi: 10.1016/S1474-4422(06)70411-2

Sofic, E., Riederer, P., Heinsen, H., Beckmann, H., Reynolds, G. P., Hebenstreit, G., et al. (1988). Increased iron (III) and total iron content in post mortem substantia nigra of parkinsonian brain. J. Neural Transm. 74, 199-205. doi: 10.1007/BF01244786

Stephens, M., Smith, N. J., and Donnelly, P. (2001). A new statistical method for haplotype reconstruction from population data. Am. J. Hum. Genet. 68, 978-989. doi: 10.1086/319501

Tanaka, G., Aminuddin, F., Akhabir, L., He, J. Q., Shumansky, K., Connett, J. E., et al. (2011). Effect of heme oxygenase-1 polymorphisms on lung function and gene expression. BMC Med. Genet. 12:117. doi: 10.1186/1471-2350-12-117

Tiroch, K., Koch, W., von Beckerath, N., Kastrati, A., and Schomig, A. (2007). Heme oxygenase-1 gene promoter polymorphism and restenosis following coronary stenting. Eur. Heart J. 28, 968-973. doi: 10.1093/eurheartj/ ehm036

Trinh, J., and Farrer, M. (2013). Advances in the genetics of Parkinson disease. Nat. Rev. Neurol. 9, 445-454. doi: 10.1038/nrneurol.2013.132

Verma, A., Hirsch, D. J., Glatt, C. E., Ronnett, G. V., and Snyder, S. H. (1993). Carbon monoxide: a putative neural messenger. Science 259, 381-384. doi: $10.1126 /$ science. 7678352

Weinreb, O., Mandel, S., Youdim, M. B., and Amit, T. (2013). Targeting dysregulation of brain iron homeostasis in Parkinson's disease by iron chelators. Free Radic. Biol. Med. 62, 52-64. doi: 10.1016/j.freeradbiomed.2013.01.017

Whitton, P. S. (2007). Inflammation as a causative factor in the aetiology of Parkinson's disease. Br. J. Pharmacol. 150, 963-976. doi: 10.1038/sj.bjp. 0707167

Yamada, N., Yamaya, M., Okinaga, S., Nakayama, K., Sekizawa, K., Shibahara, S., et al. (2000). Microsatellite polymorphism in the heme oxygenase-1 gene promoter is associated with susceptibility to emphysema. Am. J. Hum. Genet. 66, 187-195. doi: 10.1086/302729

Youdim, M. B. (2003). Rasagiline: an anti-Parkinson drug with neuroprotective activity. Expert Rev. Neurother. 3, 737-749. doi: 10.1586/14737175.3.6.737 
Zeevalk, G. D., Razmpour, R., and Bernard, L. P. (2008). Glutathione and Parkinson's disease: is this the elephant in the room? Biomed. Pharmacother. 62, 236-249. doi: 10.1016/j.biopha.2008.01.017

Zhou, C., Huang, Y., and Przedborski, S. (2008). Oxidative stress in Parkinson's disease: a mechanism of pathogenic and therapeutic significance. Ann. N.Y. Acad. Sci. 1147, 93-104. doi: 10.1196/annals.1427.023

Conflict of Interest Statement: The authors declare that the research was conducted in the absence of any commercial or financial relationships that could be construed as a potential conflict of interest.

Received: 19 June 2014; accepted: 04 September 2014; published online: 24 September 2014.
Citation: Ayuso P, Martínez C, Pastor P, Lorenzo-Betancor O, Luengo A, JiménezJiménez FJ, Alonso-Navarro H, Agúndez JAG and García-Martín E (2014) An association study between Heme oxygenase-1 genetic variants and Parkinson's disease. Front. Cell. Neurosci. 8:298. doi: 10.3389/fncel.2014.00298

This article was submitted to the journal Frontiers in Cellular Neuroscience.

Copyright (C) 2014 Ayuso, Martínez, Pastor, Lorenzo-Betancor, Luengo, JiménezJiménez, Alonso-Navarro, Agúndez and García-Martín. This is an open-access article distributed under the terms of the Creative Commons Attribution License (CC BY). The use, distribution or reproduction in other forums is permitted, provided the original author(s) or licensor are credited and that the original publication in this journal is cited, in accordance with accepted academic practice. No use, distribution or reproduction is permitted which does not comply with these terms. 